\title{
Evolution of a Small Angle Boundary from a Perfect Lattice During Annealing of a Cross Rolled Aluminium Sample
}

\author{
M. Shamsuzzoha \\ School of Mines and Energy Development, University of Alabama, Tuscaloosa, AL 35487
}

Pertinent to dislocation theory, a small angle symmetric boundary is considered to be a plane array of dislocations existing within a crystal. Atomic structure reported for low angle boundaries $[1,2]$ also describes such boundaries in terms of a regular array of dislocations separated by a nearly perfect lattice. A symmetric grain boundary of either a small angle or a high angle variety is considered to be formed by the union of two crystals along a common plane surface. The crystals across the interface after unification undertake certain arrangements of their atoms in the vicinity of boundary to match the normal crystal lattice as closely as possible. In such undertakings dislocations are formed at the positions where lattice matching occurs at the interface. Elastic continuum existing within the participating crystals allows these dislocations to be separated equally or almost equally from each other along the entire boundary as shown in a schematic of a symmetric small angle boundary of Figure 1.

In recrystallization of deformed materials small angle boundaries may also form by methods that are not involved in the unification of two crystals discussed above. During annealing lattice dislocations move in their glide plane by various methods such as glide and jumps and can pile up in groups in suitable position of the constituting crystal. Figure 2 shows such a pile-up of a group of dislocations in a crystal slab of Al prepared by cross rolling and annealing. This pile up of dislocations is likely to produce a strain field. This strain field offers collaborative interactions of dislocations and acts as a barrier to the mobility of other free dislocations in their glide planes that lie in the vicinity of the pile-up. Individual dislocation gliding in such nearby neighboring planes is likely to seize its glide in the near vicinity of such a pile up. The edge dislocations marked by arrows near locations A and B in Figure 2 might be such dislocations that stop gliding by this method. This interaction of dislocations between those pinned down in the pile up and those ceased gliding such as A and B is likely to initiate a small angle boundary from an arbitrary location of the crystal. In Figure 2 this location exists on the left side of the dislocation A. Elastic continuum of the lattice on the left of dislocation A offers prohibition to an equal space stacking of dislocations $\mathrm{A}, \mathrm{B}$ and the nearest dislocation $\mathrm{C}$ of the pile up and results in the variation of angle in the boundary at its initial stage of formation. This is evident in Figure 2, where the angle of the boundary near the vicinity of dislocations $\mathrm{B}$ and $\mathrm{C}$ can be seen progressively increased from $5^{\circ}$ to $8^{\circ}$. This variation of the boundary angle creates asymmetric segments of spacing between dislocations $\mathrm{A}, \mathrm{B}$ and $\mathrm{C}$. It is interesting to note that the edge dislocation character of these dislocations (A, B and C) also makes these boundary segments to be of purely tilt type. However, some dislocations in the pile-up of the crystal slab are of mixed character. Their collaborative interactions with other dislocations of the pile-up allow the boundary to continue beyond dislocation $\mathrm{C}$ as a mixed character boundary and to assume an increase of a few more degrees in boundary mis-orientation.

References

[1]. M J. Mills, Phil. Mag. A66, (1989) 47.

[2]. M. Shamsuzzoha, David J. Smith and P. A. Deymier, Scripta Metall. 24, (1990) 1611. 


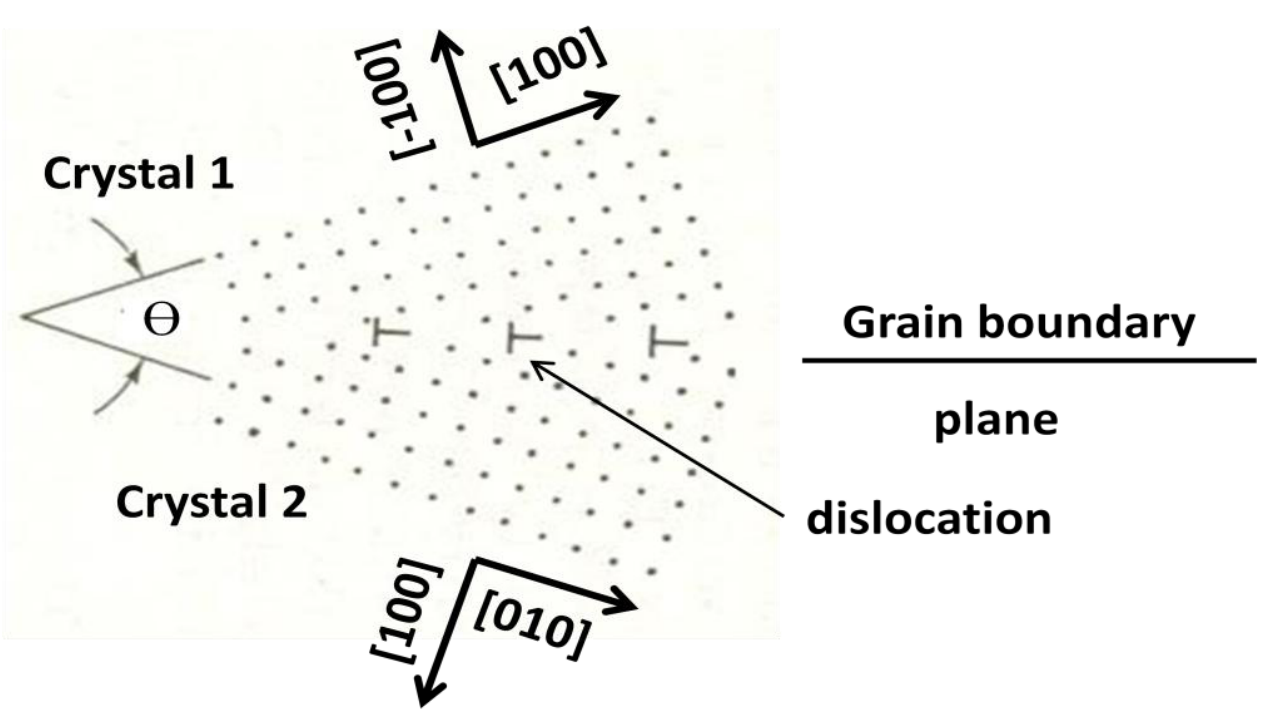

Figure 1. A schematic of the [001] projection view for a small angle grain boundary in cubic crystal.

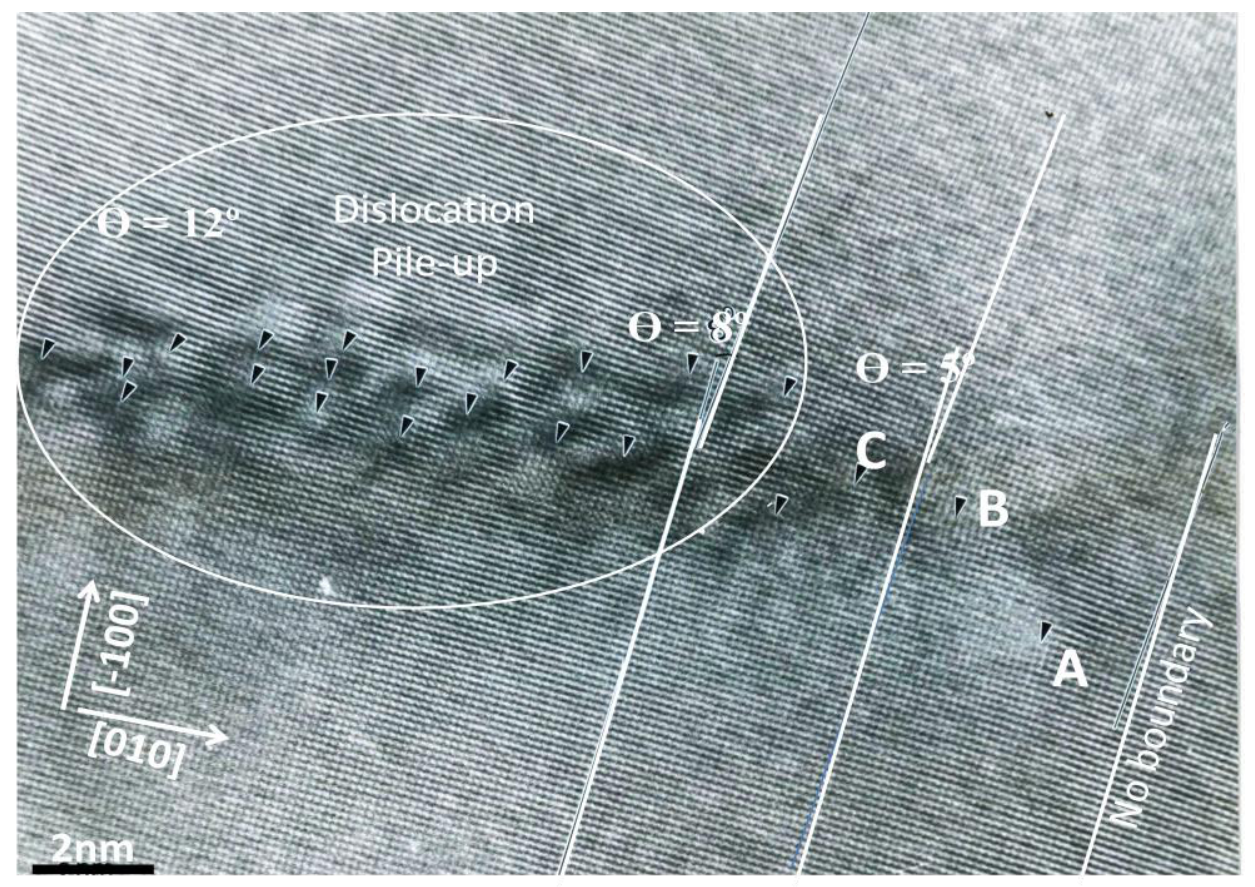

Figure 2. HREM image taken along [001] of an aluminum crystal showing how a pile up of dislocations creates a small angle boundary from the area of an otherwise perfect lattice. 